REVISTA VIRTUAL VIA INVENIENDI ET IUDICANDI

"CAMINO DEL HALLAZGO Y DEL JUICIO"

http://viei.usta.edu.co/ E-MAIL: revistainveniendi@usantotomas.edu.co

\title{
LA SITUACIÓN DE DROGODEPENDENCIA Y LA RESPUESTA DEL SISTEMA PENAL Y PENITENCIARIO ESPAÑOL: ALGUNAS CUESTIONES*.
}

\author{
Autor: \\ PROF. DRA SILVIA VALMAÑA OCHAÍTA \\ Profesora Titular de Derecho Penal \\ Facultad de Ciencias Sociales \\ Universidad de Castilla La Mancha

\section{FECHA DE RECEPCIÓN: 30 DE MARZ0 DE 2009 AUTOR INVITADO}

RESUMEN: La situación de drogodependencia genera respuestas en el sistema penal y penitenciario, de la misma manera que se producen en otros ámbitos sociales. El tratamiento de los delincuentes toxicómanos, y el análisis de las consecuencias jurídicas que encontramos relacionadas, o derivadas, de tal condición, se contemplan en el presente estudio desde la perspectiva del impacto que suponen en la individualización de la pena, y de las necesidades específicas que se plantean en el momento y durante el cumplimiento de la misma. Por una parte podemos observar que en el ordenamiento penal esta situación de drogodependencia puede producir efectos en cuanto a la culpabilidad del sujeto, y consecuencias en relación con la ejecución de la 
pena. De otra, dentro del sistema penitenciario, el tratamiento socio-sanitario del delincuente toxicómano ha ido buscando la efectividad a lo largo de los últimos años, al tiempo que se ha ido prescindiendo de algunos prejuicios a la hora de abordar la cuestión. Para conseguirlo han sido necesarias una de las legislaciones penitenciarias más modernas y respetuosa con los derechos del penado, una actuación decidida y eficaz de la Administración penitenciaria, y algunas interesantes y controvertidas resoluciones judiciales.

ABSTRACT: The situation of drug dependency generates answers in the penal and penitentiary system, in the same way as they take place in other social areas. The treatment of delinquents with drug addiction, and the analysis of the juridical consequences that we are related, or divert, of such a condition, are contemplated in the present study from the perspective of the impact that they credit with the individualization of a punishment, and from the perspective of the specific needs that appear in the moment and during the execution of a punishment. In one hand, we can observe that in the penal system, the drug addiction situation can produce effects as for the guilt of the subject, and consequences about the punishment execution. In the other hand, inside the penitentiary system, the social and sanitary treatment of the drug addict delinquent has been looking for the efficiency throughout last years. At the same time as it has been doing without some prejudices at the moment of approaching the question. To obtain these results, it has been necessary one of the most modern penitentiary legislations and respectful with the rights of the 
convicts, a determined and effective action of the Penitentiary Administration, and some interesting and controversial judicial resolutions.

PALABRAS CLAVE: Drogadicción, Prisión, Reinserción, Derecho Penal, Derecho Penitenciario.

KEY WORDS: Drug addiction, Prison, Rehabilitation, Criminal Law, Penitentiary Law.

INDICADORES JEL: K1, K3, K4

Para correspondencia con el autor:

Silvia Valmaña Ochaíta

Silvia.Valmana@uclm.es

Facultad de Ciencias Sociales de Cuenca

Seminario Permanente de Ciencias Sociales

Avda. de los Alfares, 44

16.071-CUENCA. ESPAÑA

* Este trabajo es el resultado de la investigación presentada en el Seminario Permanente de Ciencias Sociales que tuvo lugar en la Facultad de Ciencias Sociales de Cuenca (UCLM) el día 18 de febrero de 2009. Este artículo se corresponde con del Documento de trabajo, una vez revisado, que se defendió en dicho Seminario. 


\section{1.- INTRODUCCIÓN:}

La aprobación de nuestra Ley General Penitenciaria en 1979, como respuesta a la nueva concepción resocializadora de la pena, favoreció el interés por el delincuente como sujeto de estudio en toda su dimensión humana pero especialmente significado en relación con uno de los factores que ya en ese momento empezaban a mostrarse claramente asociados a la criminalidad. Me refiero al consumo de drogas y su posible potencial criminógeno, o al menos a su significativa relación en términos cuantitativos (GARCIA GARCIA, 1999). Y este interés se plasmó en estudios que abordaban la situación de drogodependencia del delincuente desde perspectivas tanto sociológicas (GARCIA MAS, 1987) como jurídicas (MEDINA MANCEBO, ESTELA BARNET, OLTRA AZNAR, GIMÉNEZ-SALINAS COLOMER, RODRÍGUEZ RODA, BERGALLI COZZI. 1990).

Los estudios que comienzan a sucederse en esta materia vinculan de manera inexorable el problema social (ESCOHOTADO ESPINOSA, 2003) y el problema penal (ELZO (Coord.), LIDON, URQUIJO, 1992) con el problema penitenciario, consecuencia inmediata y necesaria de los anteriores. La respuesta legal no se hace esperar, y es precisamente desde la misma Ley General Penitenciaria y posteriormente desde el Reglamento que la desarrolla, desde donde se abordan inicialmente dichos problemas y se sientan las bases de las soluciones (GARCÍA VALDÉS, 1983, 1986) que han llevado al penitenciarismo español a la vanguardia de la respuesta eficaz a estos retos sociales y sanitarios ${ }^{1}$.

\footnotetext{
${ }^{1}$ En el año 2006 tres proyectos españoles del ámbito de la sanidad penitenciaria fueron premiados por la Organización Mundial de la Salud y dos de ellos en relación con el tema que nos ocupa, si quiera sea
} 
En los últimos años, los problemas socio-sanitarios derivados de tráfico y del consumo de drogas han experimentado un espectacular incremento (OBSERVATORIO ESPAÑOL SOBRE DROGAS, 1998, 1999, 2000, 2001, $2002,2003,2004,2007)$, lo que ha repercutido en necesidad de una sensible mejora de los medios para el tratamiento de las situaciones de drogodependencia, al tiempo que han supuesto un endurecimiento en la represión de las conductas constitutivas de delitos contra la salud pública.

La aparición del SIDA y de las enfermedades asociadas a la infección por $\mathrm{VIH}$, ha hecho saltar las alarmas en todo el mundo en los últimos veinte años, lo que ha provocado que, sobre todo Estados Unidos, seguido de forma desigual por los países desarrollados, haya optado por una política de tolerancia cero con las conductas de tráfico de drogas, vinculando indirectamente esta política con el hecho de que la incidencia del consumo de drogas por vía parenteral en la tasa de población que padecía la infección por $\mathrm{VIH}$ era muy elevada.

indirectamente: «Protocolos de coordinación entre servicios sanitarios penitenciarios y hospitales de referencia. Infección VIH y VHC», del centro penitenciario de Fontcalent (Alicante), el primero; y a la reducción del riesgo de infección con el Proyecto premiado: «7 años del programa de intercambio de jeringuillas en la prisión de Pamplona», del centro penitenciario de Pamplona (Navarra). 


\section{Distribución de los casos de SIDA (200.531 casos) notificados la}

\section{Región Europea de la OMS hasta el 30 de septiembre de 1997}

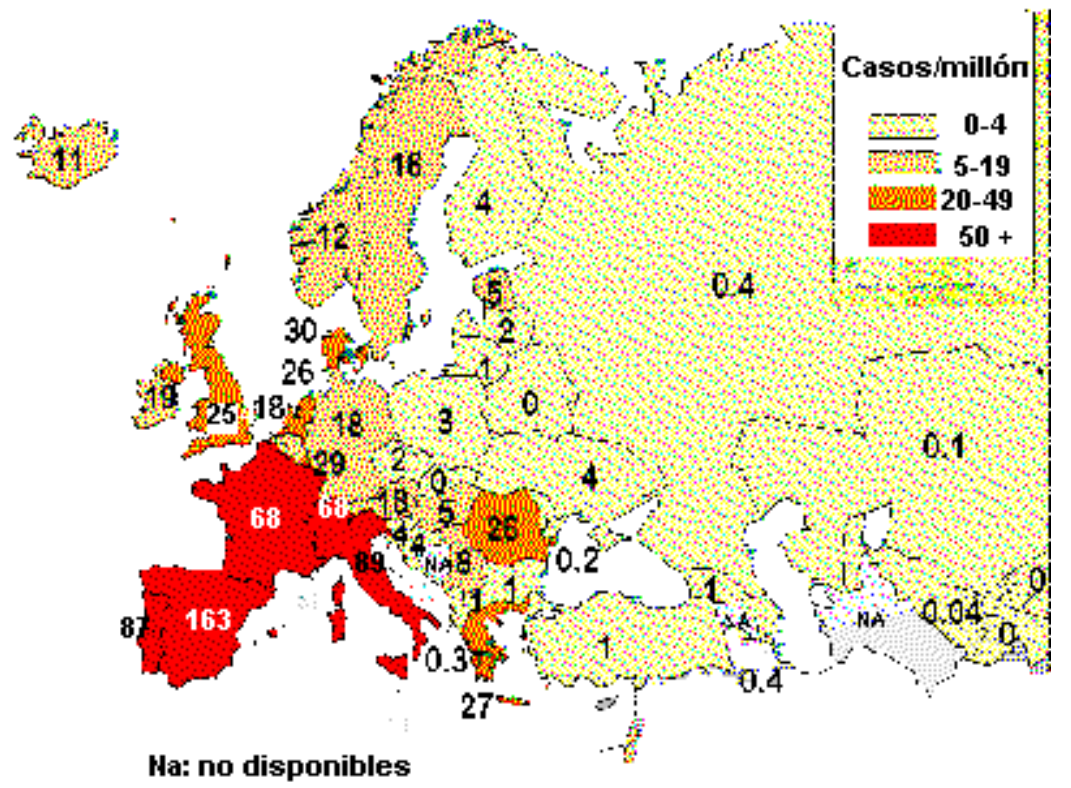

Fuente: Mapa publicado por la XII Comisión Nacional de Coordinación y Seguimiento de Programas de Prevención del SIDA (Plan de Movilización Multisectorial frente al VIH/SIDA 1997-2000. Diciembre 1997)

Y estas cifran se confirman en los más recientes estudios acerca de la población reclusa consumidora de drogas. Así "tanto los expertos penitenciarios como los responsables de la formulación de políticas reconocen que, a pesar de todas las medidas aplicadas para reducir su suministro, las drogas llegan hasta la mayoría de las prisiones. Estudios realizados entre 2001 y 2006 en Europa muestran que entre el $1 \%$ y el $56 \%$ de los reclusos declara haber consumido drogas dentro de la prisión y hasta un tercio de los reclusos ha consumido drogas por vía parenteral. Este hecho despierta preocupación en 
relación con la propagación potencial de enfermedades infecciosas, especialmente por el hecho de compartir el material de inyección" (OBSERVATORIO EUROPEO DE LAS DROGAS Y LAS TOXICOMANÍAS, 2008).

Por otra parte, y como cuestión previa, convendría, siquiera brevemente, establecer unos criterios mínimos en cuanto al concepto de droga y, en consecuencia, de drogodependencia, que se va a utilizar, en tanto que la terminología es variada en la literatura científica (LORA TAMAYO, 2003). El concepto de droga se puede abordar desde una perspectiva Farmacológica, Sociológica o Socio-sanitaria o Jurídica. En cualquier caso nos encontraremos con conceptos no convergentes que pueden dar lugar a una terminología confusa. Para evitar este problema, podemos aceptar el concepto unitario descriptivo de droga que nos proporciona SANCHEZ TOMÁS (2002) como "sustancia que consumida por el ser humano produce cambios en la actividad mental (percepción, emociones o juicio) o en el comportamiento". La inclusión de otros elementos en la definición tales como la dependencia o la tolerancia son más discutibles. Esto nos permite excluir del tratamiento que se pueda dar en el ámbito de estudio de este trabajo (concretamente en el penitenciario) a determinadas adicciones que, siendo sin duda un problema sanitario, no tienen cabida en el concepto drogodependencia (YAGÜE OLMOS, y CABELLO VÁZQUEZ. 2008). 


\section{2.- LA RESPUESTA PENAL:}

En España, junto con el incremento de las penas para los traficantes que se introduce tras la reforma de estos delitos por la Ley Orgánica 1/1988 de 24 de marzo, y que puede ser paradigma de esta tendencia, se trata de favorecer la deshabituación del toxicómano delincuente. En relación con este aspecto, la aprobación del nuevo Código Penal por Ley Orgánica 10/1995, de 23 de noviembre, consolida tal favorecimiento, cuestión esta en la que abunda la Ley Orgánica 15/2003, de 25 de noviembre, por la que se modifica el Código Penal de 1995, como veremos posteriormente.

El artículo 93 bis del anterior Código Penal, precedente directo del artículo 87 del Código Penal vigente, supone una revolución desde el momento en que crea un supuesto especial de suspensión condicional de la pena para un grupo restringido de delincuentes, los toxicómanos, para los cuales se establecían unas condiciones especiales de aplicación que no se correspondían con los requisitos generales para la suspensión condicional de la pena en los artículos 93 y 94 del citado cuerpo legal.

Esta fórmula es similar al adoptado por la mayoría de los ordenamientos europeos (SOLA DUEÑAS, HORMAZÁBAL MALAREE. 1986), que optan por un sistema de probation o de sursis avec mise a l'epreuve, en los que la condición principal pasa a ser el seguimiento de un tratamiento de deshabituación (MEDINA MANCEBO, et alt. 1990) 
El artículo $87 \mathrm{CP}$ establece, en su párrafo 1, que "aun cuando no concurran las condiciones 1 y 2 previstas en el artículo 81, el juez o tribunal, con audiencia de las partes, podrá acordar la suspensión de la ejecución de las penas privativas de libertad no superiores a cinco años de los penados que hubiesen cometido el hecho delictivo a causa de su dependencia de las sustancias señaladas en el número 2 del artículo 20, siempre que se certifique suficientemente, por centro o servicio público o privado debidamente acreditado u homologado, que el condenado se encuentra deshabituado o sometido a tratamiento para tal fin en el momento de decidir sobre la suspensión". Tales condiciones generales que pueden ser obviadas en el supuesto aquí contemplado consisten, como señala el art. 81 CP., en la primariedad delictiva y en que la pena impuesta no sea superior a dos años, respectivamente. Tales requisitos se suplen, en el primer caso, con la necesidad de una resolución motivada del juez o tribunal para establecer la suspensión en los casos de reincidencia; en el segundo, la pena suspendible será aquélla inferior a cinco años, tal y como quedó fijado tras la reforma parcial del Código Penal introducida por la Ley Orgánica 15/2003, de 25 de noviembre. Esta Ley dice en su artículo único, párrafo j) que "se introducen importantes medidas tendentes a favorecer la rehabilitación de aquellos que hubiesen cometido el hecho delictivo a causa de su dependencia de drogas, alcohol o sustancias psicotrópicas. Para ello, se permite obtener el beneficio de la suspensión cuando las penas impuestas sean hasta de cinco años, y no sólo hasta tres como ocurría hasta el momento. Además, con objeto de que la medida sea eficaz, se mejora el régimen de los requisitos que ha de cumplir el condenado, del tratamiento a que ha de someterse y de su supervisión periódica. De forma 
coordinada se prevé que, cuando esté próximo el vencimiento de la medida de internamiento para tratamiento médico 0 educación especial 0 de deshabituación, se comunique al ministerio fiscal para que inste, si fuera procedente, la declaración de incapacidad ante la jurisdicción civil”.

Además de los citados requisitos generales, para que se lleve a cabo la suspensión de la pena será también necesario el cumplimiento de unas condiciones: la primera y básica de no volver a delinquir en el periodo que se señale, y que tendrá una duración mínima de tres, y máxima de cinco años; además, en el párrafo 4 del art. 87 CP. se señala la necesidad de que, "en el caso de que el condenado se halle sometido a tratamiento de deshabituación, también se condicionará la suspensión de la ejecución de la pena a que no abandone el tratamiento hasta su finalización. Los centros o servicios responsables del tratamiento estarán obligados a facilitar al juez o tribunal sentenciador, en los plazos que señale, y nunca con una periodicidad superior al año, la información precisa para comprobar el comienzo de aquél, así como para conocer periódicamente su evolución, las modificaciones que haya de experimentar así como su finalización”. El incumplimiento de alguna de estas condiciones llevará aparejada la revocación de la suspensión. "Transcurrido el plazo de suspensión sin haber delinquido el sujeto, el Juez o Tribunal acordará la remisión de la pena si se ha acreditado la deshabituación o la continuidad del tratamiento del reo. De lo contrario, ordenará su cumplimiento, salvo que, oídos los informes correspondientes, estime necesaria la continuación del tratamiento; en tal caso podrá conceder razonadamente una prórroga del plazo de suspensión por tiempo no superior a dos años" (87,5 CP). 
Las críticas que en su momento se hicieron a la introducción del artículo 93 bis, fundamentalmente por la discriminación que suponía a favor del delincuente drogodependiente (VALMAÑA OCHAÍTA. 1990), pueden, sin embargo, revisarse en función de los resultados prácticos conseguidos con la articulación legal de lo que hoy en día supone la derivación judicial de delincuentes toxicómanos a centros de deshabituación.

A este respecto podemos concluir que la visión ofrecida por los centros asistenciales, en relación con las tasas de abandono, es que éste es inferior entre los sujetos derivados judicialmente para la realización de un cumplimiento alternativo al ingreso en prisión, frente a los sujetos no derivados judicialmente.

Así, en el Informe número 1 del Observatorio Español sobre Drogas se establece que, sobre una muestra de 812 sujetos, estudiados entre el 1 de julio de 1.996 y el 30 de junio de 1.997 , el $55^{\prime} 7 \%$ de los centros asistenciales informa de abandonos inferiores en los casos de derivación judicial, el 38’4\%, de niveles similares, y sólo el 6’1\%, de abandonos superiores con respecto a penados no derivados judicialmente.

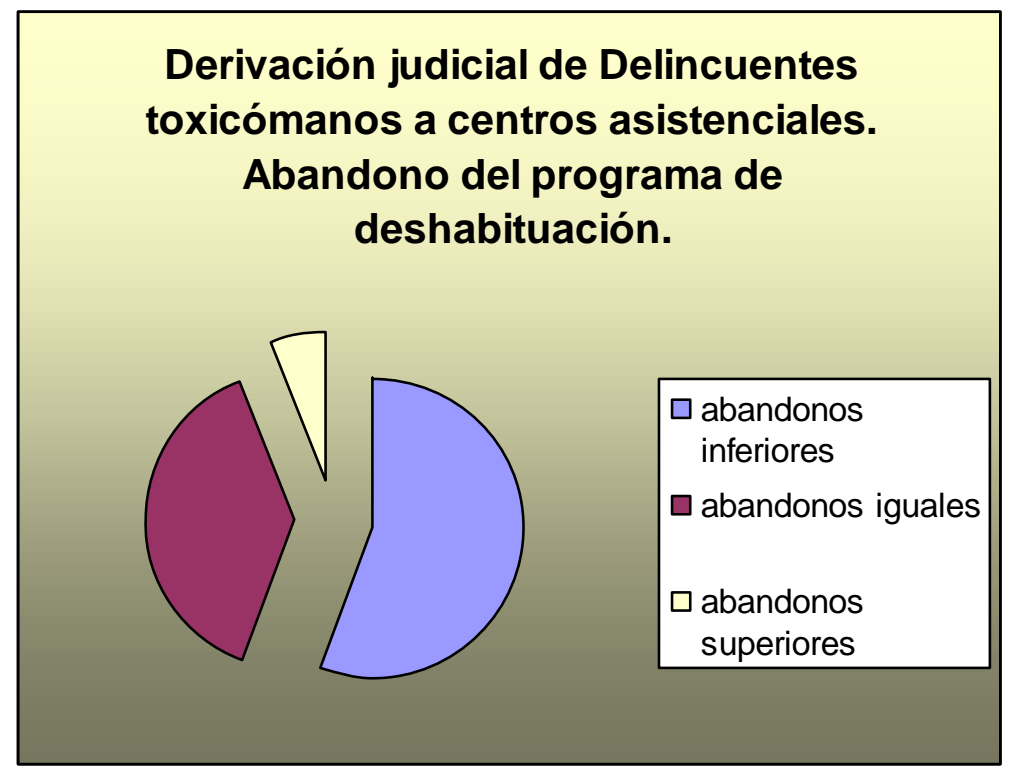

Fuente: Observatorio Español sobre Drogas. Informe n⿳⺈ 1 
El éxito de estos resultados ha incidido, sin lugar a dudas, muy directamente en el aumento de la frecuencia en la aplicación del cumplimiento alternativo de la pena privativa de libertad. Las cifras aportadas por el Observatorio Español sobre Drogas en su Informe número 4 son elocuentes: 832 personas derivadas a tratamiento en 1.999 frente a las 227 del periodo comprendido entre el 1 de junio de 1.996 y el 30 de junio de 1.997. Por su parte, el Informe número 5 (2002) establece que a "31 de diciembre del 2001, un total de 1.895 sujetos drogodependientes estaban bajo un cumplimiento alternativo en el que los tribunales sentenciadores habían requerido la intermediación de los Servicios Sociales Penitenciarios. De éstos, 1.401 sujetos estaban bajo una medida no privativa de libertad (tratamiento ambulatorio en centro sociosanitario) y 494 sujetos estaban bajo una medida privativa de libertad (internamiento en un centro de deshabituación)".

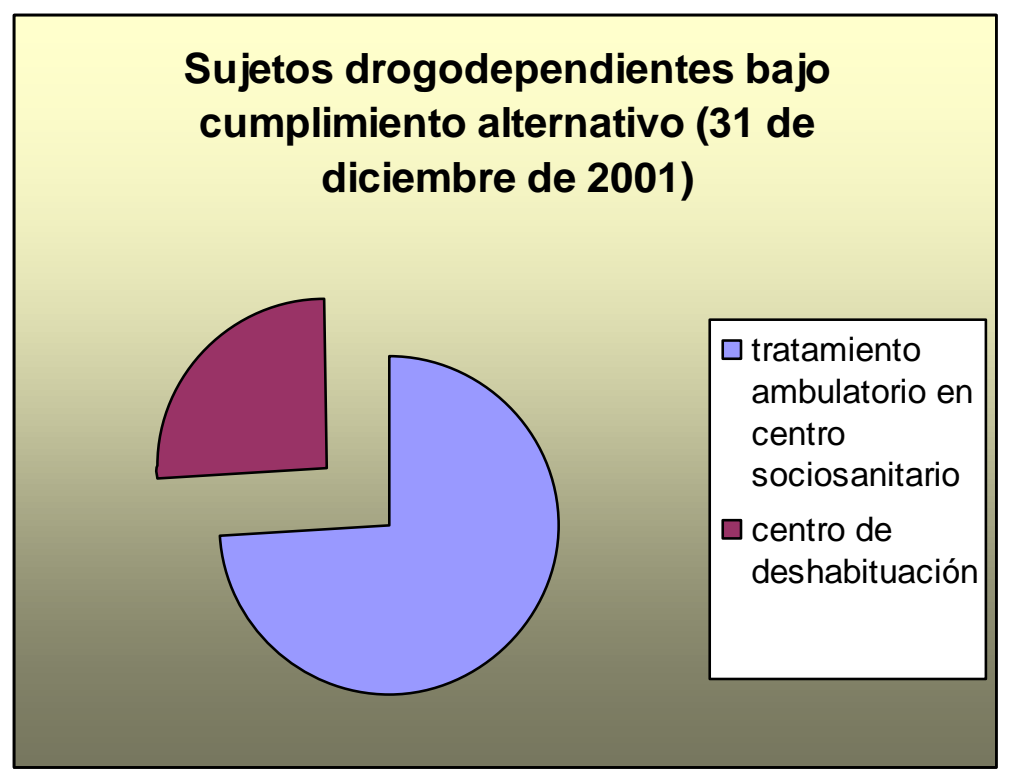

Fuente: Observatorio Español sobre Drogas. Informe ํo 5 
A pesar del paulatino e incontenible aumento que reflejan estos datos, el informe concluye que los mismos "suponen una infraestimación del número real de sujetos bajo cumplimiento alternativo, ya que los Servicios Sociales Penitenciarios no recogen todos los cumplimientos alternativos que se están aplicando en drogodependientes", en tanto que un gran porcentaje de los mismos es llevado a cabo por los Servicios de Atención al Detenido Drogodependiente en los Juzgados, que cumplen, a su vez, una misión fundamental a la hora de identificar factores de riesgo y facilitan la intervención precoz y, por tanto, mejores resultados.

Sin embargo, este cuadro se vio alterado de alguna manera con la aparición de un elemento perturbador introducido por la sentencia del Tribunal Supremo 628/2000, de 11 de abril. Esta sentencia realiza una interpretación extensiva del artículo 104 Código Penal por la cual se establece la aplicación de las medidas de seguridad previstas en el artículo 102 del Código Penal a los sujetos que, sin encontrarse en los supuestos recogidos en los artículos 102 ó 104 del Código Penal (estos es, aquellos en quienes concurran una eximente completa o incompleta de la responsabilidad de los artículos $20,2^{\circ}$ y $21,1^{\circ}$ del Código Penal respectivamente), pero a los que les sea aplicable la circunstancia atenuante del artículo $21,2^{\circ}$ del Código Penal ("la de actuar el culpable a causa de su grave adicción a las substancias en el número segundo del artículo anterior".

Dicha sentencia parte de dos criterios fundamentales: que toda adicción de larga duración a sustancias estupefacientes susceptibles de causar un grave daño a la salud, supone para el que la padece necesariamente unas graves alteraciones psíquicas, con lo que los presupuestos biológico y 
psicológico confluirían de tal manera que procedería aplicar la atenuante del artículo 21,2 del Código Penal, salvo que tal situación pudiera calificarse como eximente completa o incompleta; y que existe una laguna jurídica en lo que se refiere a la aplicación de medidas de seguridad en la atenuante de grave adicción, que puede y debe ser resuelta mediante una interpretación extensiva del artículo 104 Código Penal para, de esta forma, cumplir con la finalidad de reinserción social postulada por el artículo 25 de la Constitución (NISTAL BURÓN, 2000).

Todo ello conduce a la sentencia analizada a la conclusión de aplicar una medida de seguridad en lugar de la pena correspondiente al delito en todos los casos en que se pruebe la situación de grave adicción, si bien con la limitación de que en la imposición de la medida de seguridad "la resolución judicial debe valorar el cumplimiento de las finalidades de la pena, retribución, prevención general y especial, y las posibilidades y garantías de rehabilitación en cada caso concreto" (Fundamento de Derecho $2^{\circ}$, STS 628/2000, de 11 de abril).

Sin poner en cuestión los posibles avances que la aplicación de medidas de seguridad en lugar de penas para la reinserción de delincuentes adictos al consumo de drogas y otras sustancias que creen grave adicción pudiera tener, e incluso aceptando la conveniencia de introducir los cambios legislativos que permitieran tal aplicación, me parece sin embargo censurable la quiebra del principio de legalidad que se produce en la sentencia comentada.

Quiebra que se deriva no sólo de una interpretación extensiva de un precepto penal, sino de una interpretación gravemente contraria a la ley penal. La propia sentencia comentada reconoce las diferentes vías que le legislador 
establece para la reinserción del delincuente toxicómano y que van desde la aplicación de alternativas como la suspensión de la ejecución de las penas privativas de libertad del artículo 87 del Código Penal (siempre y cuando la pena suspendida no sea superior a 3 años), la atenuación de la pena para el sujeto con grave adicción (artículo 21,2ํㅡㄹ del Código Penal), la aplicación de medidas de seguridad cuando concurrieren eximentes completas o incompletas (artículo 104 del Código Penal), hasta la posibilidad de cumplimiento de la pena privativa de libertad con tratamiento de deshabituación intrapenitenciario o incluso en instituciones extrapenitenciarias (artículo 182 del Reglamento Penitenciario).

Y sin embargo, asume, a través de una interpretación al menos cuestionable, una competencia que no lo es propia cual es transformar una pena en una medida de seguridad, en un caso que no ha sido contemplado por la ley. Si los Tribunales de Justicia siguen "rellenando lagunas" allí donde lo crean necesario el papel del legislador deviene irrelevante incluso en el ámbito penal en el que la reserva de ley opera con mayor rigor (VALMAÑA OCHAÍTA. 2003). 


\section{3.- LA RESPUESTA PENITENCIARIA:}

Pero la cuestión fundamental de este trabajo es la observación de la realidad penitenciaria propiamente dicha, realidad que corre paralela a la penal y que se integra en el nuevo concepto socio-político al que hacía referencia al comienzo del estudio.

Desde los años 80, La Dirección General de Instituciones Penitenciarias ha reconocido el consumo de drogas en las prisiones españolas de forma expresa, llegando a señalarse que entre el $60 \%$ y el $90 \%$ de los reclusos lo había hecho en algún momento, incluyendo en estos datos el consumo no habitual. La respuesta a esta realidad suponía, casi exclusivamente, una mayor vigilancia y control de los reclusos y de las instalaciones para evitar la entrada de substancias prohibidas, así como la represión, no sólo administrativa, sino también penal, de tales conductas. Sin embargo, es justo afirmar que la función resocializadora de la pena privativa de libertad estaba presente desde el primer momento de la entrada en vigor de la Ley Orgánica 1/1.979, de 26 de septiembre, General Penitenciaria, materializando la reinserción por la vía de la deshabituación a través de los recursos, escasos, entonces disponibles: el Hospital General Penitenciario y las unidades especiales de los Centros Penitenciarios (GARCÍA VALDÉS, 1983, 1986).

Este nuevo impulso al tratamiento de drogodependientes se encontraba muy condicionado en la práctica por la insuficiencia y la deficiencia de los medios materiales para llevar a cabo tal función, heredados de los habilitados para la aplicación de la Ley 16/1.970, de 4 de agosto, sobre Peligrosidad y Rehabilitación Social. 
Los avances que la entrada en vigor de la Ley Orgánica General Penitenciaria de 1.979, y el Reglamento que la desarrolla (R.D. 1.201/1.981, de 8 de mayo) suponen, no se manifiestan en los años siguientes en consonancia con las expectativas que habían generado dichos cuerpos legales.

Hasta bien avanzada la década de los 90 no es sino cuando, ante los problemas originados por las enfermedades infecciosas asociadas al consumo de drogas $(\mathrm{VIH}$, hepatitis $\mathrm{B}$ y tuberculosis, fundamentalmente), y con el empuje de la aprobación de un nuevo Reglamento Penitenciario (R.D. 190/1.996, de 9 de febrero), se inician medidas concretas tendentes a atajar la transmisión de dichas enfermedades en la prisiones (como los programas de intercambio de jeringuillas) y de favorecer el incremento del número de reclusos sometidos a tratamiento de deshabituación, tanto dentro del sistema penitenciario como derivados a recursos externos al mismo.

Este cambio en la percepción del problema determina que, también dentro del ámbito de la prisión, los recursos se vayan adaptando progresivamente a las necesidades. En esta línea se enmarca el Protocolo de colaboración suscrito por los Ministerios de Interior y Sanidad el día 12 de febrero de 2005, para mejorar la atención integral de la población penitenciaria drogodependiente y fomentar su reinserción sociolaboral, y que posteriormente se ha ido concretando en diferentes Convenios de Colaboración entre el Ministerio del Interior y las Comunidades Autónomas ${ }^{2}$ con el mismo fin; también conviene destacar el Convenio firmado el 2 de julio de 2003 por los

\footnotetext{
${ }^{2}$ Por ejemplo, vid. Resolución 7 de septiembre de 2005, de la Secretaría General Técnica, por la que se dispone la publicación del Acuerdo sectorial en materia de drogodependencias entre la Consejería para la Igualdad y Bienestar Social de la Junta de Andalucía y el Ministerio del Interior. Resolución de 22 de enero de 2004, de la Secretaría General Técnica, por la que se dispone la publicación del Convenio de Colaboración entre el Ministerio del Interior y la Consejería de Sanidad y Bienestar Social de la Ciudad de Ceuta, sobre Drogodependencias y Sida en el Centro Penitenciario de Ceuta. (BOE 11-02-2004).
} 
Ministerios de Interior y Sanidad para la prevención del VIH/Sida en centros penitenciarios; o la más reciente Resolución de 5 de diciembre de 2008, de la Secretaría General de Sanidad, por la que se publica el Convenio de colaboración entre el Ministerio de Sanidad y Consumo y los departamentos de Salud y Justicia de la Generalitat de Cataluña, para la puesta en marcha y la evaluación de actividades para prevenir la infección por VIH en centros penitenciarios (BOE 06-01-2009).

Si bien es cierto que los datos que se ofrecen sobre la incidencia del consumo de drogas dentro de las prisiones no son del todo fiables, en tanto que depende en buena medida de la veracidad de los autoinformes, así como de las respuestas ofrecidas a las Encuestas que se han ido realizando a lo largo de los últimos años, no obstante podemos señalar que, en función de las cifras aportadas en los Informes del Observatorio Español sobre Drogas, alrededor del $50 \%$ de la población reclusa española es consumidora de drogas. Asimismo, los informes del Observatorio Europeo señalan oscilaciones entre un porcentaje de reclusos consumidores de drogas ilegales inferior a una tercera parte en los casos de Rumania, Hungría y Bulgaria, a más del $80 \%$ de las internas de una prisión de mujeres en el Reino Unido, situándose la media de la mayoría de los países integrantes de la Unión Europea algo por encima del 50\% (OBSERVATORIO EUROPEO DE LAS DROGAS Y LAS TOXICOMANÍAS, 2008), más o menos en los mismos niveles que hemos observado para España a través de la Encuesta sobre Salud y consumo de Drogas a los Internados en Instituciones Penitenciarias (ESDIP) del año 2006. 


\section{Características sociodemográficas, penales y salud de los}

\section{internos inyectores y no inyectores de drogas (\%)}

\begin{tabular}{|c|c|c|}
\hline & INYECTOR & $\begin{array}{l}\text { NO } \\
\text { INYECTOR }\end{array}$ \\
\hline Características sociodemográficas & & \\
\hline Hombre & 94,5 & 91,4 \\
\hline Español & 95,6 & 60,2 \\
\hline Máximo nivel de estudios completado & & \\
\hline Estudios de Segundo Grado o superiores & 50,9 & 57,0 \\
\hline Situación laboral principal & & \\
\hline Trabajando & 46,2 & 75,6 \\
\hline Alojamiento previo al ingreso & & \\
\hline Hogar familiar & 76,9 & 84,8 \\
\hline Prisión & 12,4 & 8,7 \\
\hline Historial penal penitenciario & & \\
\hline $\begin{array}{l}\text { Antecedentes de ingresos en centros de } \\
\text { menores }\end{array}$ & 23,2 & 10,2 \\
\hline $\begin{array}{l}\text { Antecedentes de ingresos en centros } \\
\text { penitenciarios }\end{array}$ & 78,0 & 30,4 \\
\hline Delito que motivo el actual ingreso en prisión & & \\
\hline Delitos contra la propiedad & 74,7 & 31,3 \\
\hline
\end{tabular}




\begin{tabular}{|l|c|c|}
\hline Delitos contra la salud pública & 15,8 & 39,1 \\
\hline Delitos contra las personas & 20,9 & 23,6 \\
\hline Situación procesal & & \\
\hline Penado & 90,0 & 73,4 \\
\hline Preventivo & 10,0 & 26,6 \\
\hline Clasificación penitenciaria (1) & & \\
\hline Primer grado & 1,6 & 3,0 \\
\hline Segundo grado & 80,5 & 72,6 \\
\hline Tercer grado & 10,7 & 13,1 \\
\hline Salud Autopercibida & & \\
\hline Muy buena/buena & 51,3 & 69,1 \\
\hline Regular/mala/muy mala & 48,7 & 30,9 \\
\hline Estado serológico frente al VIH & & \\
\hline Prevalencia prueba del SIDA y conoce & & \\
\hline
\end{tabular}

(1) Cálculo efectuado sobre el total de los penados.

(2) Cálculo efectuado sobre el grupo que se había realizado el test

e informa de los resultados (prevalencia autoinformada) 
Fuente: Tabla publicada por la Delegación del Gobierno para el Plan Nacional sobre Drogas. Encuesta sobre Salud y consumo de

Drogas a los Internados en Instituciones Penitenciarias (ESDIP), 2006, pág. 80

Estas alarmantes cifras han tenido como respuesta la actuación en el ámbito penitenciario en tres tipos de intervención.

En primer lugar se sitúan los programas de desintoxicación que se ofertan a los reclusos que ingresan en prisión con drogodependencia activa y no están sometidos a otro programa de deshabituación. Con este programa de desintoxicación se pretenden evitar los efectos nocivos que para la salud del drogodependiente pueda tener la obligada y repentina abstinencia en el consumo de drogas, incardinándose, por lo tanto, en la atención sanitaria del recluso. Suelen desarrollarse, por lo tanto, en la enfermería del centro penitenciario, y su duración es breve, suficiente únicamente para paliar el impacto de la suspensión del consumo y el consiguiente síndrome de abstinencia.

En segundo lugar se encuentran los programas de "reducción del daño" y que fundamentalmente consisten en el programa de mantenimiento con metadona y el programa de intercambio de jeringuillas (ZULAICA ARISTI, 1996)

Sin duda ninguna, el programa más extendido es el del tratamiento con metadona, programa que se ha implantado en la totalidad de los Centros Penitenciarios y que ha pasado de atender a 6.606 pacientes en 1996, con una implantación de programas de mantenimiento en el $99 \%$ por ciento de los 
Centros Penitenciarios (XII COMISIÓN NACIONAL DE COORDINACIÓN Y SEGUIMIENTO DE PROGRAMAS DE PREVENCIÓN DEL SIDA ), a 11.605 reclusos en agosto de 1.997, lo que supone duplicar la cifra en sólo un año, y a 21.851 reclusos de programas penitenciarios, y 4.681 más en programas externos, en 1.999.

Por su parte, el programa de intercambio de jeringuillas, introducido en agosto de 1.977 a través de una experiencia piloto en el Centro Penitenciario de Bilbao (FERNÁNDEZ LARRINOA, V., 1999), ha ido paulatinamente creciendo en cuanto al número de Centros que disponen de este programa y, por lo tanto, en el numero de usuarios del mismo, hasta alcanzar los 1.005 reclusos en 1.999. A partir de 2001, y en relación con la "contrastada" viabilidad real del programa de intercambio de jeringuillas, desde la Dirección General de Instituciones Penitenciarias se establece la Instrucción 5/ 2001 SP mediante la cual se determina que la "extensión del programa se llevará a cabo de manera paulatina, según la planificación elaborada por la Subdirección General de Sanidad. En este sentido, será esta Unidad quien comunique a cada establecimiento cuando deberá iniciarse el programa. A partir de ese momento, y con el fin de posibilitar la ejecución del mismo, se modificará la norma de régimen interior de tal modo que quede expresamente permitida la posesión de jeringuillas en los términos contemplados en el programa oficial, sin que ello suponga la modificación de ninguna de las otras normas que se hallen vigentes. Así mismo, se dará cuenta al Juzgado de Vigilancia Penitenciaria de los acuerdos del Consejo de Dirección correspondientes" (DIRECCIÓN GENERAL DE INSTITUCIONES PENITENCIARIAS, 2001). 
La idea que subyace en la aplicación de dicho programa de intercambio de jeringuillas no es otra que la de que "las personas privadas de libertad tienen derecho a disfrutar de los mismos tratamientos y programas preventivos disponibles en el medio exterior" (MENOYO MONASTERIO, C.. y BOLEA LAGUARTA, A., (Coords.). 2000), idea que aparece formulada como imperativo a cumplir por la Administración penitenciaria a partir del Auto no 247 de 14 de octubre de 1996, de la Audiencia Provincial de Pamplona viendo el Recurso de apelación contra el Auto del Juzgado de Vigilancia Penitenciaria de Pamplona, de fecha 31 de enero de 1996.

Este importante Auto venía a desestimar el Recurso de Reforma planteado frente a la resolución que resolvía la queja presentada ante dicho Juzgado por la prohibición de introducir jeringuillas en prisión y la solicitud de que se dictara una resolución por la que "se ordenase a la Dirección de la Prisión Provincial de Pamplona la puesta en práctica, en colaboración con la administración autonómica o estatal competente o con Organizaciones no gubernamentales, de un programa de prevención con intercambio de jeringuillas; (...) Tal solicitud fue denegada por el Juzgado de Vigilancia Penitenciaria, estimando que carecía de competencia, según lo dispuesto en el art. 76 de la L.O.G.P. y concordantes de su reglamento". Salvada en principio la cuestión formal con la estimación de que la queja ante el Juzgado sería el instrumento adecuado para plantear la cuestión suscitada, el Auto referido entra en el fondo del asunto sobre la base de los siguientes argumentos:

1.- La infección por VIH puede transmitirse por compartir agujas o jeringuillas contaminadas en casos de autoadministración por vía intravenosa de drogas. 
2.- Se consumen drogas por vía intravenosa dentro de la prisión, intercambiando los internos para ello agujas y jeringuillas.

3.- La OMS, en sesión celebrada en Ginebra en 1987, realizó una serie de recomendaciones encaminadas a evitar el contagio del VIH en las prisiones, entre las que destaca la necesidad de aplicar en las prisiones los mismos principios adoptados por los Programas Nacionales sobre el SIDA para el resto de los ciudadanos, debiendo reconocer la Administración penitenciaria su responsabilidad para minimizar la transmisión del VIH en prisión y establecer las medidas precisas para ello.

4.- El art. 3,4 de la L.O.G.P. establece que "la actividad penitenciaria se ejercerá respetando, en todo caso, la personalidad humana de los recluidos y los derechos e intereses jurídicos de los mismos no afectados por la condena, (...)" y que "La administración penitenciaria velará por la vida, integridad y salud de los internos".

5.- Esta acreditado el establecimiento de "un programa de intercambio de agujas y jeringuillas usadas mediante la entrega del Kit Antisida elaborado por el Gobierno de Navarra, de uso habitual por parte de las personas usuarias de drogas por vía parenteral".

6.- Las medidas preventivas no son incompatibles con las normas internas de seguridad, ni con el hecho de que el consumo de drogas en prisión sea considerado una falta grave, porque tales medidas de prevención se adoptan "para evitar el contagio y la transmisión de enfermedades que puedan contraerse en relación con prácticas asociadas a la conducta sancionada”.

El Auto analizado, ante las consideraciones precedentes, concluye estableciendo que "la Dirección de la Prisión Provincial de Pamplona deberá 
poner en práctica un programa de prevención con intercambio de jeringuillas como prestación de la Administración Penitenciaria mediante las medidas de asistencia previstas en los arts. 207 y ss del Reglamento Penitenciario, bien directamente o a través de los convenios, conciertos o contratos que considere oportuno, (art. 212) o bien en el ámbito de la dispensación farmacéutica y prestaciones complementarias básicas (art. 209).

Por último precisar como ya se ha dicho que la indicada medida de prevención está destinada restringidamente a los reclusos toxicómanos, sin que se trate de un medio de favorecimiento al consumo, pudiendo la administración penitenciaria establecer las medidas de control que considere precisas para asegurar un uso adecuado de la aguja y la jeringuilla, que por lo tanto podrán ser objeto de requisa si concurriesen circunstancias que los hiciesen aconsejable".

Además del impulso que sin duda esta Auto supuso al inicio del programa de intercambio de jeringuillas, conviene señalar que la iniciativa del mismo corresponde al Parlamento Vasco que, en su función de control e impulsos políticos, aprobó por acuerdo del pleno de 13 de diciembre de 1995 la Proposición no de Ley por la que se instaba al Gobierno Vasco a solicitar a la Secretaría de Estado de Asuntos Penitenciarios que con carácter experimental, y bajo los correspondientes controles sanitarios y de seguridad, ponga en marcha un programa piloto de intercambio de jeringuillas en un de los tres centros penitenciarios, destinado a un grupo reducido de internos/as, cuya evaluación permita, en su caso, adoptar una decisión uniforme y razonada para la introducción de jeringuillas en el medio penitenciario". En la misma línea se produce la iniciativa que, a raíz de una proposición no de Ley del Partido 
Socialista, modificada en la Comisión Mixta para el Estudio del Problema de las Drogas, en su sesión de 20 de marzo de 2001, acuerda instar al Gobierno para que, "en el plazo de tiempo más breve posible, adopte todas las medidas que resulten necesarias y en especial implante el programa de distribución gratuita de jeringuillas entre los reclusos toxicómanos, a fin de evitar en lo posible la propagación entre los mismos de enfermedades contagiosas por vía hemática tales como la hepatitis o el sida"

A partir de ese momento, con programas experimentales primero (AGUIRRE ESUNZA et alt. 1999), hasta culminar con el Programa Marco de Intercambio de jeringuillas en el medio penitenciario (MINISTERIO DEL INTERIOR, 2002) se ha venido contrastando el éxito de las medidas adoptadas en la prevención del contagio del $\mathrm{VIH}$ y otras enfermedades asociadas al consumo de drogas por vía intravenosa.

\section{Programa de Intercambio de Jeringuillas en prisiones por}

\section{Comunidad Autónoma. España 2005}

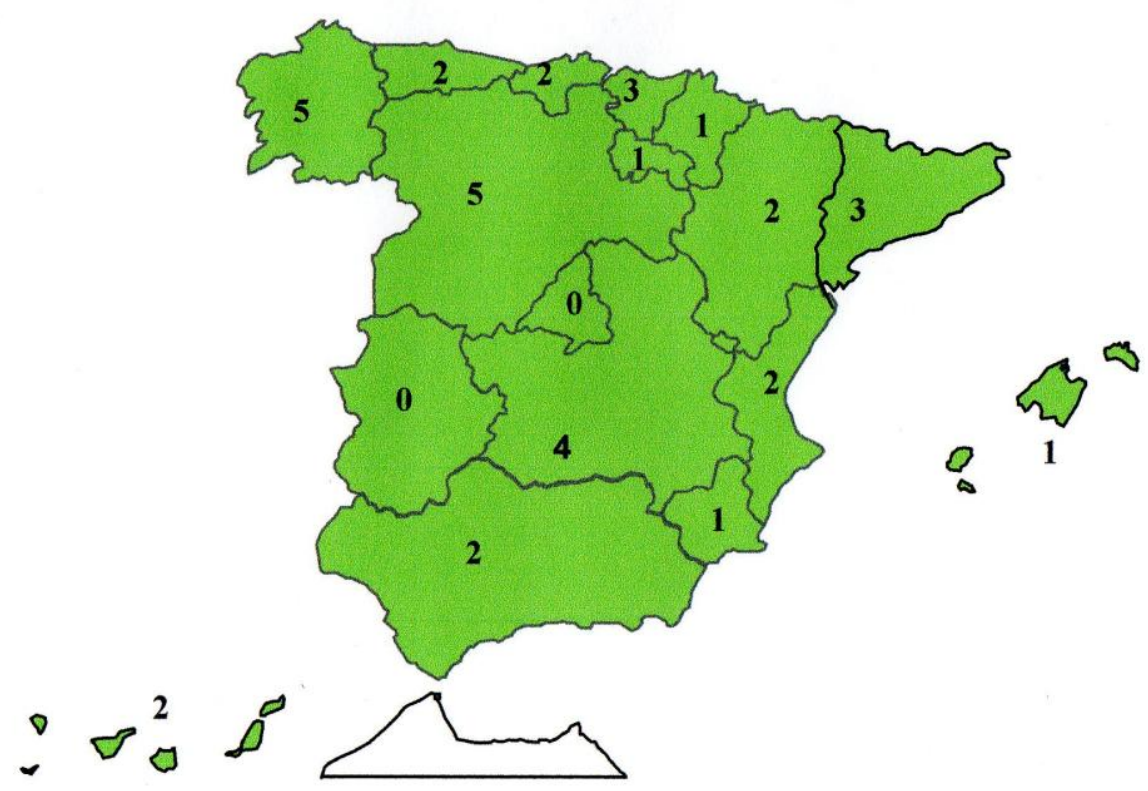


Fuente: Mapa publicado por el Ministerio de Sanidad y Consumo.

Directorio del Programa de Intercambio de Jeringuillas en Prisiones. 2005

Por último, el tercer bloque de actuaciones lo integran los programas de deshabituación, que a su vez pueden ser desarrollados en los Centros Penitenciarios o bien a través de la derivación de reclusos a recursos externos.

Dentro de estos programas intrapenitenciarios, cabe destacar por la importancia del número de sujetos atendidos, el tratamiento ambulatorio, en el que el sujeto recibe tratamiento deshabituador sin ser separado del resto de la población reclusa, frente al tratamiento modular, en el que el sujeto es atendido en un módulo específico, que a su vez puede ser utilizado como centro de día, o módulo terapéutico, si también pernoctan en el mismo, alcanzándose de este modo el mayor grado de separación del resto de los internos.

En cuanto a la derivación de los reclusos a recursos externos, contemplada en el artículo 182 del Reglamento Penitenciario de 1.996, ésta permite la asistencia del penado, clasificado en tercer grado, en instituciones terapéuticas adecuadas, públicas o privadas, para recibir un tratamiento específico de deshabituación de drogodependencias, siempre y cuando la institución de acogida apruebe un programa de deshabituación que incluya el compromiso expreso de la misma de acoger al interno, así como de comunicar al Centro Penitenciario las incidencias que surjan en el tratamiento; asimismo, el interno debe consentir y comprometerse expresamente a llevar el régimen de vida que le sea impuesto en la institución de acogida, y aceptar el programa de 
seguimiento aprobado conjuntamente por el Centro Penitenciario y la institución, de forma expresa y previa al inicio del tratamiento.

La utilización de estos recursos externos de manera complementaria a las actuaciones intrapenitenciarias ha ido aumentando de forma progresiva en los últimos años. Así, frente a los 4.553 internos en programas de mantenimiento con metadona en 1.998, en 1.999, el número se situó en 4.861 beneficiarios de este servicio en programas comunitarios; de los 2.519 sujetos en tratamiento ambulatorio de 1.998 , se ha pasado a los 2.847 en 1.999; en cuanto a los que ingresaron por esta vía en comunidades terapéuticas, se ha alcanzado la cifra de 615 en 1.999, frente a los 502 de 1.998 (datos ofrecidos por el Observatorio Español sobre Drogas: Informe 4).

Esta sería a grandes rasgos, la realidad de los drogodependientes condenados a penas privativas de libertad hoy en España, y la descripción del paulatino aumento de las fórmulas que facilitan el tratamiento y deshabituación de los mismos, y que se desarrollan en el ámbito penitenciario español a través de los programas intrapenitenciarios o de derivación a recursos externos.

\section{4.- BIBLIOGRAFÍA:}

- AGUIRRE ESUNZA, JA, BADIOLA SANTAMARIA, JA, BARCELO, F, BURÓN BENGOETXEA, M, CONDE ZABALA, MJ, FERNÁNDEZ DE LARRINOA PALACIONS, V, GABANTXO LAKA, K, GARRIDO SECO, GA, LÓPEZ BLANCO, JA, MARTÍN ZURIMENDI, JA, MARTÍNEZ LEUNDA, JA, MENOYO MONASTERIO, C, PARRAS VÁZQUEZ, F, RUIZ FERNÁNDEZ, J, SAIZ RUIZ DE LOIZAGA, F, ZULAICA ARISTI, D. El Programa de Intercambio de 
Jeringuillas de la prisión de Basauri (Bizkaia) (1997-99). Recuperado el 27 de marzo de 2009, de

http://www.msc.es/ciudadanos/enfLesiones/enfTransmisibles/sida/prevencion/p rogInterJeringuillas/PIJPrisiones/pijEvaluacion.htm

- CORTES GENERALES, "Acuerdo adoptado por la Comisión Mixta para el Estudio del Problema de las Drogas, en su sesión de 20 de marzo de 2001, de aprobar con modificaciones la Proposición no de Ley por la que se solicita la distribución gratuita de jeringuillas en los centros penitenciarios entre los reclusos toxicómanos (núm. Expíe. 161/390), presentada por el Grupo Parlamentario Socialista". 161/000390 (CD); 663/000006 (S). BOGC. Cortes Generales, VII Legislatura, Serie A, núm. 119, 28 de marzo de 2001, pág. 2.

- DELEGACIÓN DEL GOBIERNO PARA EL PLAN NACIONAL SOBRE DROGAS. Encuesta sobre Salud y consumo de Drogas a los Internados en Instituciones Penitenciarias (ESDIP), 2006. ). Recuperado el 27 de marzo de 2009 de

http://www.pnsd.msc.es/Categoria2/publica/pdf/encuestaPenitenciaria2006.pdf

- DIRECCIÓN GENERAL DE INSTITUCIONES PENITENCIARIAS, Instrucción 5/ 2001 SP, de 7 de junio. 2001. Recuperado el 27 de marzo de 2009 de http://www.mir.es/INSTPEN/INSTPENI/Archivos/c-2001-05.pdf

- ELZO, J., (Coord.), LIDON, JM., URQUIJO, ML., Delincuencia y drogas. Análisis jurídico y sociológico de sentencias emitidas en las audiencias provinciales y en los juzgados de la C.A.V. Servicio Central de Publicaciones del Gobierno Vasco. 1992 
- ESCOHOTAdO ESPINOSA, A., Historia y sociología de las drogas, En Drogodependencia y Derecho, IBAÑEZ SOLAZ, MF (Dir.), Cuadernos de Derecho Judicial. 2003

- FERNÁNDEZ DE LARRINOA, V., Programa piloto de intercambio de jeringuillas en el centro penitenciario de Basauri. Revista de servicios sociales, ำ. 36, 1999.

- GARCIA GARCIA, J., Drogodependencias y Justicia penal, Ministerio de Justicia y Ministerio del Interior. 1999

- GARCIA MAS, MP (Dir.), La droga en una cárcel de mujeres, Consejería de Salud y Bienestar Social de la Comunidad de Madrid. Instituto Regional de Estudios (IRES), 1987.

- GARCÍA VALDÉS, C., La droga y la Institución penitenciaria, Universidad de Alcalá de Henares, 1983.

- GARCÍA VALDÉS, C., Droga e Institución penitenciaria. Droga y privación de libertad. Depalma, 1986.

- LORA TAMAYO, C., Aspectos farmacológicos de las drogas (Concepto, terminología y clasificación. Los procesos de obtención. Las bases bioquímicas. Farmacología). En Drogodependencia y Derecho, IBAÑEZ SOLAZ, MF (Dir.), Cuadernos de Derecho Judicial. 2003

- MEDinA MANCEBo, O., Estela baRnet, A., OltRA AZNAR, J., GIMÉNEZ-SALINAS COLOMER, S., RODRÍGUEZ RODA, J., BERGALLI COZZI, V. Drogodependencias y sistemas penal y penitenciario en Holanda, Repúublica Federeal de Alemania, Suecia, Italia, Francia y Escocia. Colección INTRESS. Serie Estudios 2. 1990 
- MENOYO MONASTERIO, C. y BOLEA LAGUARTA, A., (Coords.) Elementos clave para la implantación de Programas de Intercambio de Jeringuillas en Prisión. (2000). Recuperado el 27 de marzo de 2009 de http:/www.msc.es/ciudadanos/enfLesiones/enfTransmisibles/sida/prevencion/p rogInterJeringuillas/PIJPrisiones/elemClavePIJ.htm

- MINISTERIO DEL INTERIOR. Intercambio de jeringuillas en el medio penitenciario. Programa Marco. 2002. Recuperado el 27 de marzo de 2009 de http://www.msc.es/ciudadanos/enfLesiones/enfTransmisibles/sida/prevencion/a ctividadesEnPrisiones/docs/programaMarco.pdf

- MINISTERIO DE SANIDAD Y CONSUMO, Directorio PIJ prisiones, 2005. Recuperado el 27 de marzo de 2009 de http://www.msc.es/ciudadanos/enfLesiones/enfTransmisibles/sida/prevencion/a ctividadesEnPrisiones/img/mapaPIJPrisiones2005.pdf

- NISTAL BURÓN, J., El tratamiento de los delincuentes toxicómanos: comentarios a la Sentencia del Tribunal Supremo 628/2000, de 11 de abril, Revista de Estudios Penitenciarios, no 248, 2000.

- OBSERVATORIO ESPAÑOL SOBRE DROGAS. Informe número 1. 1998. Recuperado el 27 de marzo de 2009 de http://www.pnsd.msc.es/Categoria2/publica/pdf/oed-1.pdf

- OBSERVATORIO ESPAÑOL SOBRE DROGAS. Informe número 2. 1999. Recuperado el 27 de marzo de 2009 de http://www.pnsd.msc.es/Categoria2/publica/pdf/oed-2.pdf

- OBSERVATORIO ESPAÑOL SOBRE DROGAS. Informe número 3. 2000. Recuperado el 27 de marzo de 2009 de http://www.pnsd.msc.es/Categoria2/publica/pdf/oed-3.pdf 
- OBSERVATORIO ESPAÑOL SOBRE DROGAS. Informe número 4. 2001. Recuperado el 27 de marzo de 2009 de http://www.pnsd.msc.es/Categoria2/publica/pdf/oed-4.pdf

- OBSERVATORIO ESPAÑOL SOBRE DROGAS. Informe número 5. 2002. Recuperado el 27 de marzo de 2009 de http://www.pnsd.msc.es/Categoria2/publica/pdf/oed-5.pdf

- OBSERVATORIO ESPAÑOL SOBRE DROGAS. Informe número 6. 2003. Recuperado el 27 de marzo de 2009 de http://www.pnsd.msc.es/Categoria2/publica/pdf/oed-6.pdf

- OBSERVATORIO ESPAÑOL SOBRE DROGAS. Informe 2004. Recuperado el 27 de marzo de 2009 de http://www.pnsd.msc.es/Categoria2/publica/pdf/oed-6.pdf - OBSERVATORIO ESPAÑOL SOBRE DROGAS. Informe 2007. Recuperado el 27 de marzo de 2009 de http://www.pnsd.msc.es/Categoria2/publica/pdf/oed-2007.pdf - OBSERVATORIO EUROPEO DE LAS DROGAS Y LAS TOXICOMANÍAS. EI problema de la drogodependencia en Europa. Informe anual 2008. Recuperado el 27 de marzo de 2009 de http://www.pnsd.msc.es/Categoria3/coopera/pdf/Informe2008.pdf

- PARLAMENTO VASCO, Proposición no de Ley referente a distribución de jeringuillas en centros penitenciarios de Euskadi, formulada por el Grupo Parlamentario Izquierda Unida/Ezker Batua/Berdeak (Acuerdo del Pleno de 13 de diciembre de 1995) (05/11.02.01.0051). Parlamento Vasco. Boletín Oficial, oㅜ 52, V Legislatura, 29.12.1995, págs. 2457 y 2458 
- SANCHEZ TOMÁS, JM., Derecho de las drogas y las drogodependencias, Fundación de Ayuda contra la Drogadicción. 2002.

- SOLA DUEÑAS, A., HORMAZÁBAL MALAREE, H. Alternativas a la prisión. Editorial PPU. 1986.

- VALMAÑA OCHAÍTA, S., Sustitutivos penales y proyectos de reforma en el Derecho Penal español, Ministerio de Justicia, 1990.

- VALMAÑA OCHAÍTA, S., Drogodependencia y relaciones de especial sujeción, en Derecho y prisiones hoy, LEÓN VILLALBA, J. (Dir.). Universidad de Castilla La Mancha, 2003.

- XII COMISIÓN NACIONAL DE COORDINACIÓN Y SEGUIMIENTO DE PROGRAMAS DE PREVENCIÓN DEL SIDA, Plan de Movilización multisectorial frente al VIHISIDA 1997-2000, Madrid, 19 de diciembre de 1997. Recuperado el 27 de marzo de 2009 de http://www.msc.es/ciudadanos/enfLesiones/enfTransmisibles/sida/planesEstrat/ planMultisectorial.htm

- YAGÜE OlmOS, C., y CABELlO VÁZQUEZ, M.I., Programa de deshabituación tabáquica para internos y trabajadores de un centro penitenciario, Revista Española de Sanidad Penitenciaria. 2008

- ZULAICA ARISTI, D. (Coord.), Guía para la puesta en marcha de programas de intercambio de jeringuillas. Subcomisión de prevención. Plan Nacional sobre el sida. 1996. Recuperado el 27 de marzo de 2009 de http://www.msc.es/ciudadanos/enfLesiones/enfTransmisibles/sida/docs/tbc.pdf 